\title{
HEALTH BENEFITS OF STRAWBERRIES
}

\author{
*Dr. Divya Choudhary
}

The red colour fruit is not only delicious to eat but is packed with nutrients that offer a range of health benefits..

\section{Boosts your immunity}

Strawberries are packed with Vitamin $\mathrm{C}$, which helps boost your immunity and protects you from infections. Just one cup of strawberries meets $100 \%$ of your daily requirement of Vitamin $\mathrm{C}$.

\section{Prevents heart disease}

Shaped like a heart, strawberries have nutrients like flavonoids and antioxidants which prevent the build-up of bad cholesterol which can clog your arteries. Additionally, these nutrients have antiinflammation properties which is good for the heart.

\section{Prevents constipation and diabetes}

When you have constipation, it is important that you consume fibre in order to clear your bowel movements. Also, fibre helps regulate your blood sugar levels making it a great food for diabetics and those who want to prevent diabetes alike. Strawberries have a lowly glycemic index of 40, which means diabetics can consume it without worrying too much.

\section{Fights cancer}

Like most fruits and vegetables, strawberries too are high in antioxidants which help in fighting cancer. In particular, strawberries have antioxidants like lutein and zeathanacins which suppresses the growth of cancer cells.

\section{Good for skin}

When you age, your skin and other body parts suffer from damage due to free radicals. This process is similar to the process of a metal rusting due to exposure to air. Vitamin $\mathrm{C}$ and antioxidants present in strawberries can help slow down this process and keep your skin wrinkle-free for longer.

\section{Helps you lose weight}

Strawberries are extremely low in calories, with a cup having just 53 calories. Also, they have fibre which can leave you feeling fuller if you have them before a meal. Not only this, the high Vitamin C content help boost your metabolism which helps your body burn the calories faster.

\section{Great for pregnant woman}

Pregnant women are often confused as to what they should eat during pregnancy that'll both be good for them and the baby. Folate present in rich quantities in strawberries helps in the baby's brain, skull and spinal cord development. In addition, it also has folic acid which can save the child from having certain birth defects.

\section{Great for bones}

Strawberries have nutrients like manganese, potassium and some minerals that not only help promote bone growth, but also helps keep them in mint condition. These properties also make strawberry a good fruit for growing children.

*Professor, Kasturba Gandhi Nursing College, SBV, Puducherry. 


\section{Prevents hair loss}

Hair loss can be quite a depressing condition for anyone. Folic acid, Vitamin B5, Vitamin B6 and ellagic acid present in strawberries helps prevent your hair from falling and thinning. Furthermore, minerals like copper and magnesium helps prevent dandruff and fungal growth on your scalp. If that wasn't enough, many home made packs can be made using strawberries that'll add shine and glow to your hair.

\section{Boosts memory}

Fisetin, a naturally occurring flavonoid present in strawberries helps enhance memory by stimulating the signaling pathways. A research published in Annals of Neurology also proved that eating 2 or more servings of strawberries per week can delay memory decline in ageing women

So, why wait? Go to your nearest fruit seller and get a delicious box of strawberries home! 